\title{
What are the Limitations in the Characterization of Self-Assembled Metamaterials using Advanced Microscopy Techniques?
}

\author{
C.J. Kiely, ${ }^{*}$ M. Watanabe, ${ }^{*}$ A. Burrows, ${ }^{*}$ P. Clasen, ${ }^{*}$ M.P. Harmer, ${ }^{*}$ B.Rodríguez-González, ${ }^{* *}$ \\ L. Liz-Marzán, ${ }^{* *}$ I. Hussain, ${ }^{* * *}$ J. Fink*** and M. Brust $* * *$
}

* Center for Advanced Materials and Nanotechnology, Lehigh University, 5 East Packer Avenue, Bethlehem, PA 18015-3195, USA.

** Departmento de Quimica Fisica, Universidad de Vigo, 36200, Vigo, Spain.

*** Center for Nanoscale Science, University of Liverpool, Liverpool, Merseyside, L69 7ZD, UK.

The controlled manipulation of materials on the nanometer scale is an important task for the production and precision positioning of ever more compact electronic, optical and magnetic components. It is now possible to create thin films, nanowires and 3-D supercrystals by exploiting the order inducing chemical interactions that are inherent to a particular system. For instance, ordered structures comprised of monosized ligand stabilized nanoparticles (of most materials) can be 'self-assembled' from a solution of their components simply by evaporating a drop of such solution onto a suitable substrate. The current state-of-the-art is the production of metamaterials whereby nanoparticles of different chemical identities and sizes are self-assembled to form nanocomposite materials. Alkanethiol capped gold and silver nanoparticles were the first to be assembled in regular arrays $^{[1]}$ followed by metamaterials composed of ordered magnetic (iron oxide) and semiconducting (lead selenide) nanoparticles ${ }^{[2]}$. In principle there an infinite number of combinations of different nanoparticle types that could be self-assembled into new metamaterials, provided that in each case, the ligand shell and solvent chemistry of both species can be made compatible. This opens up a whole new vista of designer nanomaterial combinations (e.g. metals with semiconductors, nonmagnetic with magnetic metals, ceramics with polymers, etc). Each new metamaterial will display physical properties determined by the nature of the nanoparticle components and the linker ligand molecules. Characterizing the structure, composition and physical properties of such metamaterials poses significant new challenges for the materials scientist.

Consider the Au-Ag nanoalloy raft shown in Figure 1(a). Particle size and morphology, as well as the quality of the raft ordering, can to some extent be assessed by simple visible inspection of BF and ADF images. In the future however, HAADF tomography offers the possibility of directly visualizing the $3 \mathrm{D}$ facet structure of individual nanoparticles ${ }^{[3]}$. Particle and superlattice crystallography can currently be evaluated by analysis of polycrystalline ring patterns and low angle diffraction patterns respectively (Figures 1(b,c)). A future goal is to be able to perform automated CBED pattern analysis to map the relative orientations of all the particles in the array (analogous to the automation now available in EBSD). The chemical identity of the individual particles in the array can be established by EDS or EFTEM analysis (Figure 1(d)). However if the array were composed of chemically more complicated core-shell or alloy nanoparticles, then a much higher spatial resolution spectroscopic analysis would be required. For instance, Figure 2 shows STEMEDS maps of multi core-shell Au@Ag@Au nanoparticles. These maps have been enhanced using multivariate statistical analysis (MSA): a statistical method ideally suited to spectral analysis of small particles ${ }^{[4]}$. Protocols for quantitatively mapping the composition of such complex entities are only just being developed. Aberration corrected STEM affords the new possibility of performing atomic-column-by-atomic-column XEDS and EELS spectroscopy of nanomaterials ${ }^{[5]}$, allowing in 
principle for instance, the location of one dopant atom within an individual semiconductor nanoparticle. Furthermore, in order to measure physical properties such as the local electrical conductivity in such spatially confined metamaterials it will be necessary to use specialized TEM specimen rods with multiple in-built STM probes for transport measurements within the microscope. In-situ TEM heating experiments also have a key role to play in characterizing these metamaterials since their thermal stability is likely to be poorer than their bulk counterparts due melting point depression effects that occur on the nanoscale.

A final more sobering thought follows: most of the nanoparticle systems currently in vogue are ligand stabilized. Whilst we have a growing armory of techniques for studying the (usually) crystalline core, the number of methods available for characterizing the soft ligand shells is much more limited. It is vital that we should make a real effort to understand the ligand shell structure (maybe using complimentary techniques such as WAXS or EPR) as this can make up to $30-40 \%$ of the material volume in these self-assembled nanocomposite systems. Important questions to be resolved include:- What is the typical surface coverage of ligands on the nanoparticle surface? Do sufficient vacant sites exist to allow ligand exchange or diffusion on the surface? When particles are self-assembled into superstructures do individual ligands or bundles of ligands interdigitate?

\section{References}

[1] C.J. Kiely et al., Advanced Materials, 13, (2001), 1800.

[2] F. Redl, K.S. Cho, C.B. Murray and S. O’Brien, Nature, 423, (2003), 968.

[3] P. Midgley et al., Microscopy \& Microanalysis, 9(2), (2003), 4.

[4] P.G. Kotula et al., Microscopy \& Microanalysis, 9, (2003), 1.

[5] S.J. Pennycook et al, Microscopy \& Microanalysis, 9(2), (2003), 2.
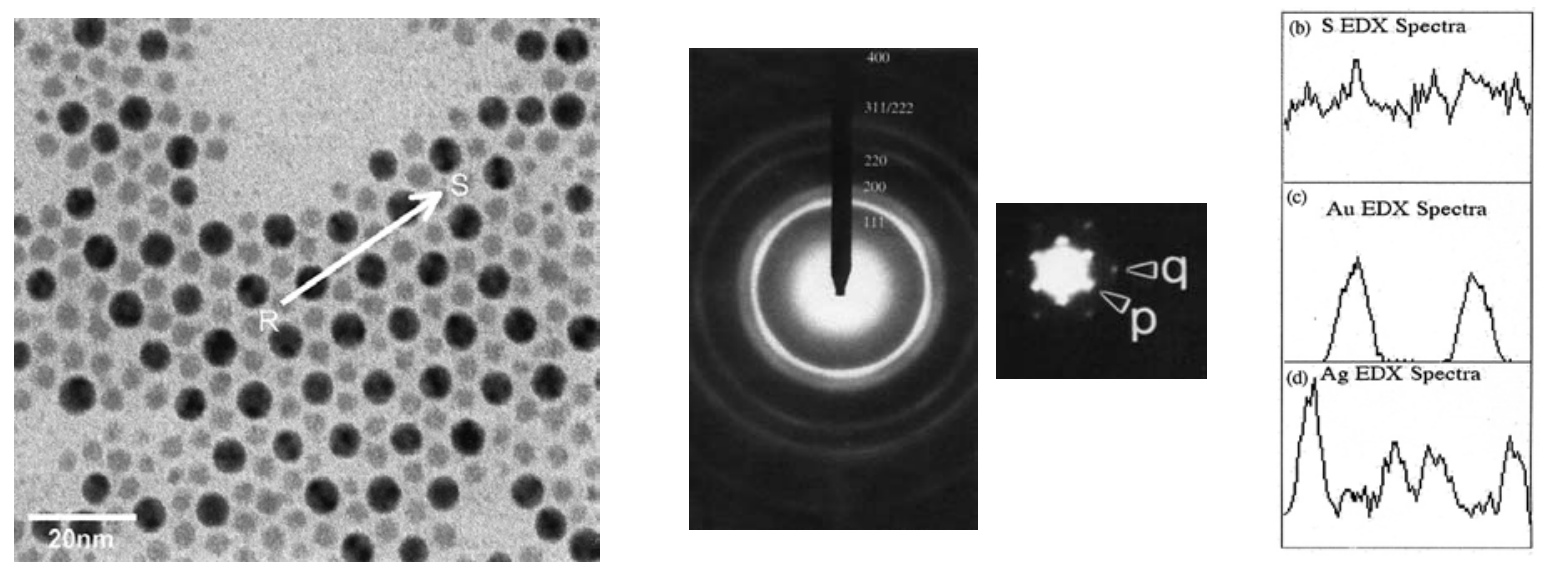

Figure 1: (a) BF micrograph showing a Au-Ag metamaterial raft: $(b, c)$ polycrystalline and low angle diffraction patterns from the Au-Ag array: (d) S, Au and Ag XEDS scans taken along RS.
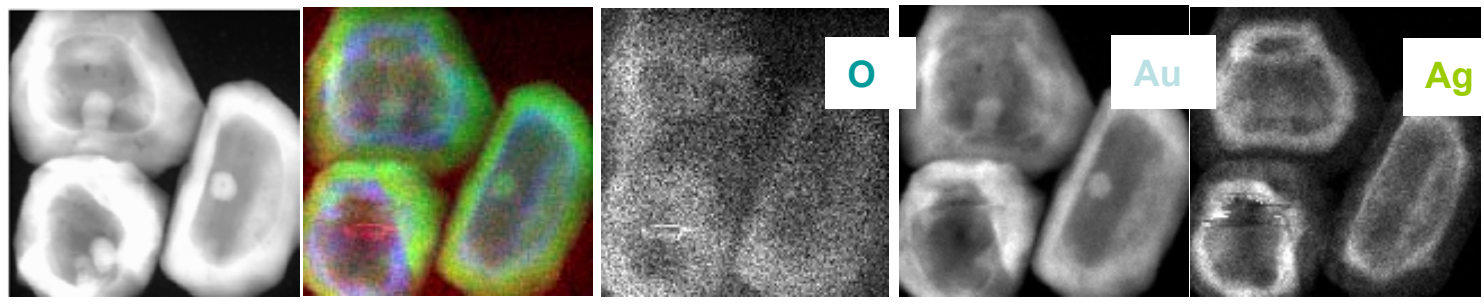

Figure 2: ADF images and XEDS maps of Au@Ag@Au multi core-shell nanoparticles. 